\title{
Comparative Study of the Mesenchymal Stem Cell and Simvastatin in the Treatment of Hepatic Fibrosis in Rats Induced by Carbon Tetrachloride
}

\author{
Ali M. Ali, Essam M. Eid, Hanan I. El-Kerdasy, Marian V. Zaki, Naglaa A. Sarg
}

Department of Anatomy and Embryology, Benha faculty of medicine, Banha University, Egypt.

Correspondence to: Hanan I. El-Kerdasy, Department of Anatomy and Embryology, Benha faculty of medicine, Banha University, Egypt.

Email:

mhanan909@yahoo.com

Received: 13 June 2021

Accepted: 27 November 2021

\begin{abstract}
Background: Hepatic fibrosis is an important medical disease with high rates of morbidity and mortality. Bone marrow mesenchymal stem cells have been recommended as a powerful therapy for treatment of liver fibrosis. Simvastatin has an ameliorative effect on fibrosis of different organs. The study aimed at comparing between the effect of BM-MSCs and simvastatin in treatment of liver fibrosis induced by carbon tetrachloride. Material and Methods: Fifty rats were categorized into, group I control group, group II in which rats injected by $\mathrm{CCl} 4$ to induce hepatic fibrosis. Group III in which rats were injected by CCL4,then injected intravenously by a single dose of BM-MSCs, group IV in which rats were injected by CCL4 then given simvastatin orally once daily for eight weeks. Group V in which rats were given both BM-MSC and simvastatin. At the end of the experimental study, blood samples were collected for biochemical analysis and liver tissues were prepared for histological and immunohistochemical examination. Results: CCL4 significantly elevated liver enzymes and induced destruction of normal
\end{abstract} hepatic structures with significant elevation of mean area percentage of collagen fibers deposition and TGF- $\beta$ expression. BM-MSC and simvastatin improved liver enzymes and histological structure of liver tissue, but the anti-fibrotic effect of BM-MSC was superior to that of simvastatin. Moreover, combination of BM-MSC and simvastatin exerted strong anti-fibrotic effect and preserved normal histologic structure of liver tissue more than each of them alone.

Conclusion: A combination of BM-MSCs and simvastatin has an ameliorative role in the treatment of liver fibrosis induced by $\mathrm{CCl} 4$.

Key words: Liver fibrosis, CCL4, BM-MSCs, simvastatin, TGF- $\beta$ 


\section{Introduction}

Liver fibrosis is a frequent consequence of chronic liver diseases, like hepatitis viral infection $\mathrm{C}$ and $\mathrm{B}$, alcoholic and non-alcoholic fatty liver disease, immune diseases including autoimmune hepatitis and exposure to pollutants or chemicals as pharmaceuticals (1). Excess extracellular matrix that is deposited in liver fibrosis distorts hepatic vasculature and impedes the exchange of oxygen and nutrient between hepatocytes and liver sinusoids that may cause cell apoptosis (2).

CCL4 is a clear liquid with sweet smell, widely used in fire extinguishers, as a precursor to refrigerants and as a cleaning agent; it is a frequent environmental pollutant. People are exposed to CCL4 through inhalation from the atmospheric environment and by dermal contact. Because of its injurious role, its uses are now excluded and only used in a number of industrial fields (3).

CCL4 causes acute liver toxicity as it lead to liver necrosis owing to the release of free radicals that generate lipid peroxide and cause alteration in enzyme activity, cell membrane damage, and lastly initiation of hepatic damage (4).

Hepatic stellate cells (HSCs) are activated all through liver fibrogenesis, additionally there is an increased inflammatory response and excessive accumulation of extracellular matrix proteins $(E C M)$ that lead to the formation of fibrous scars and destruction the normal hepatic architecture. Untreated cases progress rapidly to form liver cirrhosis (5)

Mesenchymal stem cells (MSC) are considered as a new method of treating liver fibrosis in spite of the original cause. MSCs can be isolated from deferent tissues, as well as bone marrow, umbilical cord blood and adipose tissue. Bone marrow-derived MSCs (BM-MSCs) are the simplest and has been widely studied (6). BM-MSCs are able to attenuate liver fibrosis by different mechanisms including inhibition of HSC activation, reduction of the proliferation of activated HSC, weakening the inflammatory response and enhancing the hepatocyte regeneration (7)

Statins are 3-hydroxy-3-methylglutaryl coenzyme A reductase inhibitors, which are used in the treatment of liver fibrosis. Statins lower the level of cholesterol in the blood, improve the endothelial dysfunction, and increase nitric oxide bioavailability. Moreover, it has antifibrotic, antioxidant, antiinflammatory and immunomodulatory properties (8). 
The study aimed to compare between the therapeutic effects of BM-derived MSCs or simvastatin or a combination of both in rat model of carbon tetrachloride induced liver fibrosis.

\section{Materials and Methods}

\section{Animal:}

This experimental study was done on fifty adult male albino rats weighting between 200220 gm. They were housed at the animal house unit of Faculty of Medicine, Cairo University. Rats were kept under standard conditions, suitable temperature during a period of four months from April 2019 to July 2019. Handling of these animals followed all the experimental animal committee of the national research council guidelines.

\section{Isolation and labeling of MSCs:}

Bone marrow was harvested by flushing the tibiae and femur of six week old male albino rats (other than rats of the studied groups) with Dulbecco's modified Eagle's medium supplemented with $1 \%$ penicillin streptomycin. After that cells incubated at 37 ${ }^{\circ} \mathrm{C}$ in $5 \%$ humidified $\mathrm{CO} 2$ for $12-14$ days as a primary culture. Culture was cleaned by phosphate buffer saline (PBS) and $0.25 \%$ trypsin in $1 \mathrm{mmol} /$ EDTA. Then centrifuged at 2,400 rpm for $20 \mathrm{~min}$, and suspended with serum supplemented medium. Isolated MSCs were identified by their adhesiveness and fusiform shape under light microscope. MSCs were prepared at Unit of Biochemistry and Molecular Biology, Biochemistry Department, Faculty of Medicine, Cairo University. (9).

\section{Drugs}

Carbon tetrachloride: It was obtained from El Nasr chemical industries company. Cairo. Egypt. In the form of vial $1 \mathrm{mg}$ dissolved in 1 $\mathrm{ml}$ of olive oil.

Simvastatin (Zocor): It was obtained from the local market, manufactured by Global Napi, Egypt under license of Merck \& Co, USA. In the form of tablets $10 \mathrm{mg}$ simvastatin, each tablet dissolved in $5 \mathrm{ml}$ of $0.5 \%$ solution of xanthan gum.

\section{Experimental design}

Fifty rats were included in this study; the rats were categorized into five groups:

- Group I (control group): Ten rats were equally divided into two subgroups Subgroup a: Five rats were feed on standard diet without any drugs.

Subgroup b: Five rats were injected intraperitoneally with $1 \mathrm{ml} / \mathrm{kg}$ olive oil, the solvent of CCL4twice /week for 6 weeks.

- $\quad$ Group II(carbon tetrachloride group): Ten rats were injected intraperitoneally by 
$\mathrm{CCl} 4$ at a dose of $1 \mathrm{mg} / \mathrm{kg}$ dissolved in $1 \mathrm{ml}$ of olive oil twice /week for six weeks, then sacrificed (10).

- $\quad$ Group III (BM-MSCs- treated group): Ten rats were given $\mathrm{CCl} 4$ for six weeks as in group II. After that, these rats were injected by a single dose of BM-MSCs $\left(3 \times 10^{6}\right.$ cells in 1.5 $\mathrm{ml}$ of PBS) intravenously through the tail vein. Then rats were sacrificed after eight weeks (10).

- Group IV (Simvastatin- treated group): Ten rats were given $\mathrm{CCl} 4$ for six weeks as in group II. After that, these rats were given simvastatin at a dose of $10 \mathrm{mg} / \mathrm{kg}$ by gastric tube dissolved in $0.5 \%$ solution of xanthan gum daily for eight weeks, then sacrificed (10).

- $\quad$ Group V (MSC+ Simvastatin- treated group): Ten rats were given $\mathrm{CCl} 4$ for six weeks as in group II. Then they were given both MSCs and SIMV in the same doses as in group III and IV.

At the end of the experiment rats were sacrificed by inhalation of diethyl ether. A mid line abdomeno-thoracic incision was done and blood samples were collected directly from the heart for biochemical analysis. Liver specimens were dissected and prepared for histological examination.

\section{Biochemical examination:}

The collected blood samples were centrifuged at 3,000 rpm for fifteen minutes to separate serum, for measurement of serum levels of alanine aminotransferase (ALT), aspartate aminotransferase (AST), alkaline phosphatase (ALP) and albumin(11).

\section{Histopathological examinations}

Liver samples from right lobe were fixed in $10 \%$ buffered formalin then embedded in paraffin. After that, serial sections of $5 \mu \mathrm{m}$ thickness samples were taken, and stained with hematoxylin and eosin (H\&E) and Masson's trichrome stains for detection of collagen fibers accumulation in liver tissue (12).

Transforming growth factor-beta (TGF- $\beta$ ) immunostaining:

Four $\mu \mathrm{m}$ serial sections were taken from the paraffin-embedded tissues then, dewaxed and rehydrated. Sections were then blocked with 3 $\%$ hydrogen peroxide and $10 \%$ nonimmuneanimal serum and incubated with TGF-b polyclonal antibody, and second antibody of biotin-labeled, streptavidin anti-biotin horseradish peroxidase. After that, stained with diaminobenzidine and Mayer hematoxylin, then dehydrated in xylene. The positive expression of TGF-b in the liver 
tissue was confirmed by the presence of brown-deposited granules (13).

Immunohistochemical stain (CD 105)

CD105 immunostaining was used for detection of MSCs markers in hepatic tissue treated with BM-MSC. Primary antibody for CD105 rabbit polyclonal Ab (ab27422) (14).

\section{Morphometric study:}

Mean area percentage of collagen fibers accumulation and TGF- $\beta$ immunostaining were measured in ten images from all groups using Image-Pro Plus program version 6.0 (Media Cybernetics Inc., Bethesda, Maryland, USA).

\section{Statistical analysis:}

All collected data obtained from the study were recorded and analyzed using One-way analysis of variance (ANOVA) with Post Hoc LSD test applied to evaluate the differences and comparison between all groups.

\section{Results:}

Biochemical examination: (table 1)

Serum AST, ALT and ALP levels showed significant increase ( $\mathrm{p}<0.05)$ and serum albumin level was significantly decreased $(\mathrm{p}$ $<0.05$ ) in group II in comparison with control group. However, there were a significant reduction ( $\mathrm{p}<0.05)$ in AST, ALT and ALP levels and a significant increase $(p<0.05)$ in serum albumin in groups III, IV and $\mathrm{V}$ in comparison to group II. Moreover, those levels were better in group $\mathrm{V}$ than those in group III\& IV.

\section{Histopathological Examination}

H\&E stained liver sections from subgroup a \& $\mathrm{b}$ in the control group were comparable and showed normal polygonal classic hepatic lobule. Hepatocytes were radiating from the central vein toward the periphery of the lobule, hepatocytes are with vesicular nuclei and acidophilic cytoplasm, some hepatocytes are binucleated, blood sinusoids are in between hepatocytic cords (fig 1A). Normal portal area contained portal vein and bile duct, hepatocytes with vesicular nuclei and acidophilic cytoplasm, some hepatocytes are binucleated and blood sinusoid is in between hepatocytic cords (fig 1B).

On the other hand, liver sections from group II showed congested dilated central vein, wide congested sinusoid. Hepatocytes have vacuolated cytoplasm and marked inflammatory cell infiltration (fig 2A). Most hepatocytes have vacuolated cytoplasm others with deep pyknotic nucleus (fig 2B). The portal area contained dilated congested portal vein, with inflammatory cell infiltration. Most hepatocytes have vacuolated cytoplasm, others with deep pyknotic nucleus (fig 2C). While, in 
group III there was central vein, numerous hepatocytes with vesicular nucleus, some hepatocytes are binucleated, few with vacuolated cytoplasm and dilated blood sinusoid open in the central vein (fig 3A). Portal area with portal vein, few inflammatory cell infiltrations, numerous hepatocytes with vesicular nucleus, some hepatocytes are binucleated, few with vacuolated cytoplasm and blood sinusoids (fig 3B)

The liver sections from group IV showed congested central vein, wide sinusoid, hepatocytes with vesicular nucleus, and others were binucleated, some with vacuolated cytoplasm (fig 4A). Portal area contained congested portal vein, wide sinusoid, inflammatory cell infiltration, hepatocytes with vesicular nucleus, other hepatocytes with pyknotic nucleus and some with vacuolated cytoplasm (fig 4B).

However, liver sections from group V showed nearly normal hepatic tissue, the hepatocytes were radiating from the central vein, hepatocytes had vesicular nuclei and acidophilic cytoplasm, some hepatocytes were binucleated and sinusoids were in between hepatocytic cords (fig 5A). Portal area contained portal vein; hepatocytes with vesicular nuclei and acidophilic cytoplasm, some hepatocytes are binucleated and normal blood sinusoids (fig 5B)
Masson trichrome stained liver sections from the control group showed scanty collagen fiber deposition around portal area (fig 6A). While that in group II showed marked collagen fibers deposition around portal area (fig 6B). However, group III showed mild collagen fibers deposition around portal area (fig 6C). And in group IV, there was moderate amount of collagen fiber deposition around portal area (fig 6D), and a little collagen fiber deposition around the portal area in group $\mathrm{V}$ (fig 6E).

\section{TGF-b1immunostaining:}

TGF-b1 immunostaining of liver sections from the control group showed scanty immune-positive reaction (fig 7A). While immunostaining in group II showed a high positive immune reaction (fig 7B). But in group III, it showed a mild reaction (fig 7C). Section in group IV showed moderate reaction (fig 7D). However, in group V, there was a little immune-positive reaction (fig 7E).

\section{105 immunostaining:}

Immunohistochemical detection of CD105 positive stem cells in liver section from control group showed negative reaction for CD105 antibody (fig 8A). On the other hand, sections from groups III and V showed a positive reaction for CD105 antibody (fig 8B\& 8C) indicating presence of stem cell. 


\section{Morphometric study:}

Mean area percentage of collagen fiber deposition in CCL4 group was significantly increased $(\mathrm{P}<0.01)$ in comparison with that in control group. However, there was a significant decreased $(\mathrm{P}<0.01)$ in III, IV and $\mathrm{V}$ groups in comparison with that in CCL4 group. While, it was significantly decreased in group V compared with that in II, III and IV groups (Table 2) (fig 6F).
The mean area percent of TGF $b$ immune positive expression in CCL4 group was significantly increased $(\mathrm{P} \quad 0.01)$ in comparison with that in control group. While, in groups III, IV and V there was a significant decreased $(\mathrm{P}<0.01)$ in comparison with that in CCL4 group. Moreover, it was significantly decreased in group V compared with that in II, III and IV groups (Table 3) (fig 7F).

Table (1): Serum levels of AST, ALT, ALP and Albumin in groups I, II, III, IV and V

\begin{tabular}{ccccc}
\hline Group & AST (U/L) & ALT(U/L) & ALP (U/L) & $\begin{array}{c}\text { Albumin } \\
(\mathbf{m g} / \mathbf{d l})\end{array}$ \\
\hline I & $100.55 \pm 0.44$ & $46.45 \pm 1.5$ & $130.68 \pm 0.6$ & $3.47 \pm 0.08$ \\
II & $245.73 \pm 0.97^{\mathrm{a}}$ & $111.57 \pm 2.3^{\mathrm{a}}$ & $285.68 \pm 0.86^{\mathrm{a}}$ & $2.25 \pm 0.1^{\mathrm{a}}$ \\
III & $135.85 \pm 0.62^{\mathrm{ab}}$ & $74.35 \pm 0.84^{\mathrm{ab}}$ & $185.55 \pm 0.62^{\mathrm{ab}}$ & $2.93 \pm 0.14^{\mathrm{ab}}$ \\
IV & $188.28 \pm 1.1^{\mathrm{abc}}$ & $91.1 \pm 0.53^{\mathrm{abc}}$ & $218.67 \pm 0.93^{\mathrm{abc}}$ & $2.58 \pm 0.12^{\mathrm{abc}}$ \\
V & $116.58 \pm 0.74^{\mathrm{abcd}}$ & $55.85 \pm 1.1^{\mathrm{abcd}}$ & $148.97 \pm 0.71^{\mathrm{abcd}}$ & $3.03 \pm 0.15^{\mathrm{abcd}}$ \\
\hline
\end{tabular}

Values are expressed as means $\pm \mathrm{SD}$, Value $\mathrm{p}<0.05$ is significant. ${ }^{\mathrm{a}} \mathrm{p}<0.05$ compared with Group I (control group); ${ }^{b} \mathrm{p}<0.05$ compared with Group II (CCL4 group); ${ }^{\mathrm{p}}<0.05$ compared with Group III (MSCs-treated group); ${ }^{\mathrm{d}} \mathrm{p}<0.05$ compared with Group IV (simvastatin-treated group).

Table (2): The mean area $\%$ and SD of collagen fibers deposition in groups I, II, III, IV and V

\begin{tabular}{cccccc}
\hline & Group I & Group II & Group III & Group IV & Group V \\
\hline Mean & $0.18 \%$ & $5.24 \%$ & $1.77 \%$ & $2.56 \%$ & $0.25 \%$ \\
SD & 0.0407 & 0.8591 & 0.1328 & 0.6159 & 0.0434 \\
$\begin{array}{c}\text { Significance } \\
\text { at P }<0.01\end{array}$ & Groups $2,3,4$ & Groups 1,3,4,5 & Groups & & Groups \\
& & & $1,2,4,5$ & Groups 1,2,3,5 & $2,3,4$ \\
\hline
\end{tabular}

1=sig. with group I $\quad 2=$ sig. with group II $\quad 3=$ sig. with group III $\quad 4=$ sig. with group IV $\quad 5=$ sig. with group V 
Table (3): The mean area \% and SD of TGF- $\beta 1$ immunostaining in groups I, II, III, IV and V

\begin{tabular}{cccccc}
\hline & Group I & Group II & Group III & Group IV & Group V \\
\hline Mean area \% & $0.39 \%$ & $7.12 \%$ & $1.78 \%$ & $2.71 \%$ & $0.53 \%$ \\
SD & 0.0455 & 0.4731 & 0.1571 & 0.1792 & 0.0851 \\
$\begin{array}{c}\text { Significance at } \\
\mathbf{P}<\mathbf{0 . 0 1}\end{array}$ & Groups & Groups & Groups & Groups & Groups \\
& $2,3,4$ & $1,3,4,5$ & $1,2,4,5$ & $1,2,3,5$ & $2,3,4$ \\
\hline
\end{tabular}

1=sig. with group I $\quad 2=$ sig. with group II $\quad 3=$ sig. with group III $\quad 4=$ sig. with group IV $\quad 5=$ sig. with group V

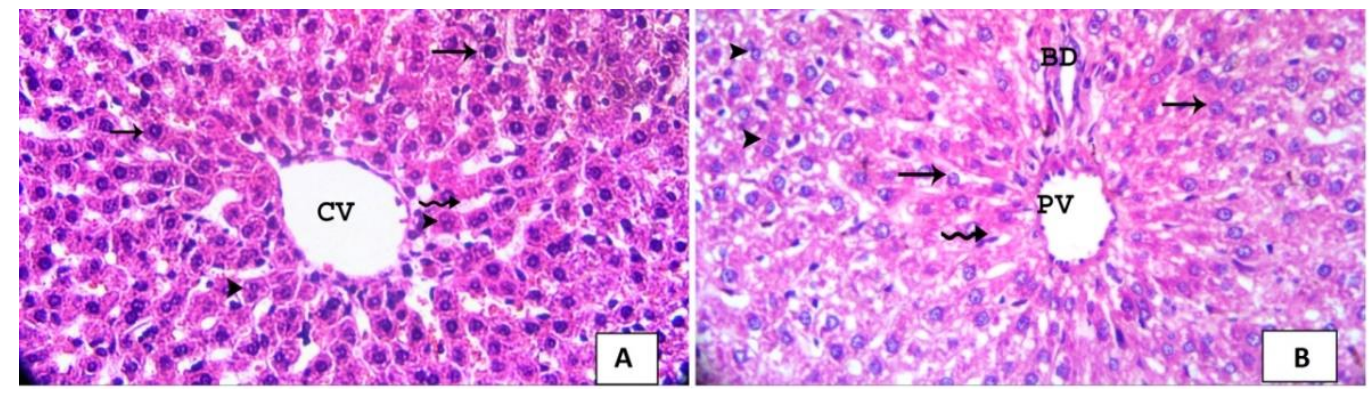

Figure (1) photographs of liver sections from the control group showing, A: Normal polygonal classic hepatic lobule, hepatocytes are radiating from the central vein $(\mathrm{CV})$ toward the periphery of the lobule, hepatocytes are with vesicular nuclei and acidophilic cytoplasm (arrows), some hepatocytes are binucleated (head arrows), blood sinusoids are in between hepatocytic cords (wavy arrows). B: Normal portal area contain portal vein (PV) and bile duct (BD), hepatocytes are with vesicular nuclei and acidophilic cytoplasm (arrows), some hepatocytes are binucleated (head arrows), blood sinusoids are in between hepatocytic cords (wavy arrows). (H\&E X 400)

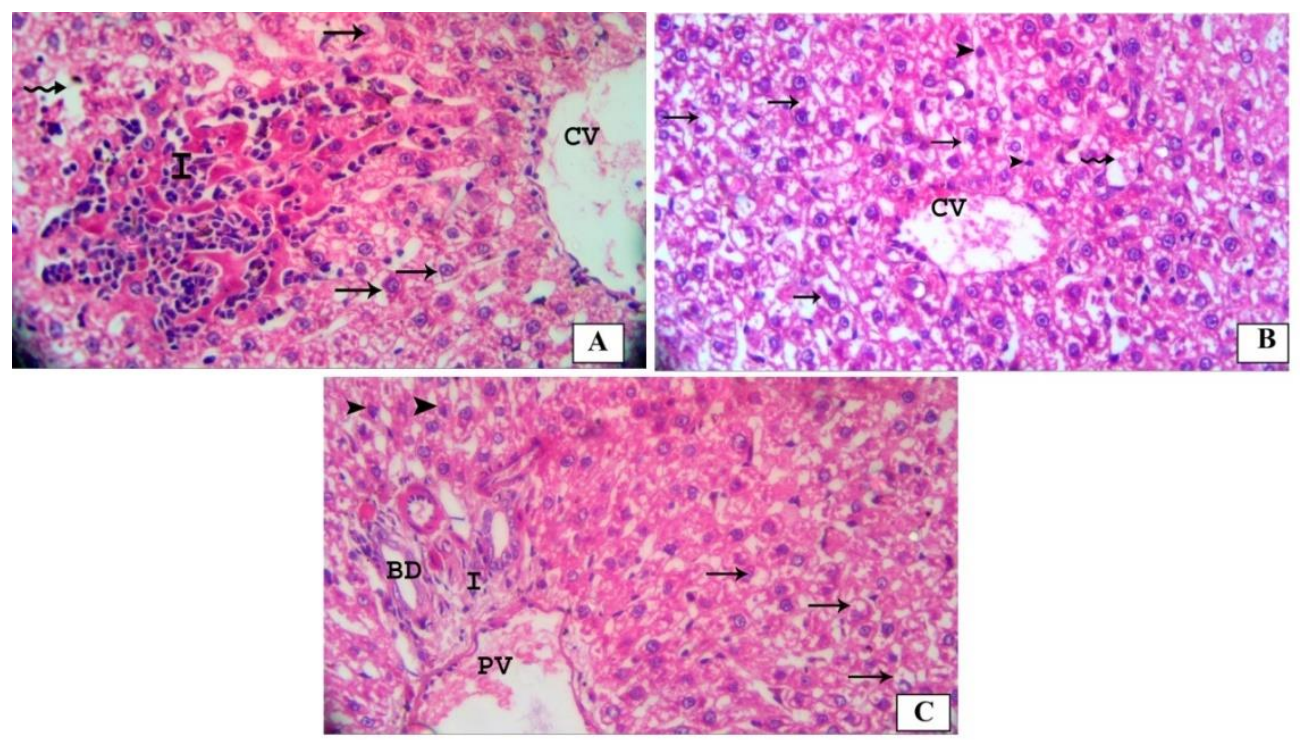

Figure (2) photographs of liver sections from CCL4 group showing, A: congested dilated central vein (CV), wide congested sinusoid (wavy arrow). hepatocytes have vacuolated cytoplasm (arrow) and marked inflammatory cell infiltration (I). B: congested central vein (CV), wide congested sinusoid (wavy arrow). Most hepatocytes have vacuolated cytoplasm (arrow), others with deep pyknotic nucleus (head arrow) C: portal area contains dilated congested portal vein (PV), bile duct (BD), with inflammatory cell infiltration (I). Most hepatocytes have vacuolated cytoplasm (arrow) and others with deep pyknotic nucleus (head arrow) (H\&E X 400) 

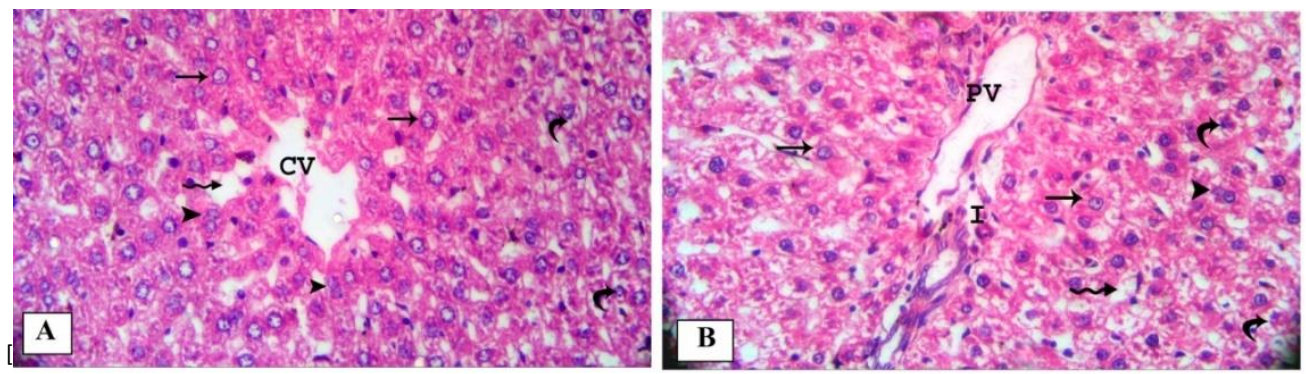

Figure (3) photographs of liver sections from MSCs - treated group showing, A: central vein (CV), numerous hepatocytes with vesicular nucleus (arrows), some hepatocytes are binucleated (head arrows), few with vacuolated cytoplasm (curved arrow) and dilated blood sinusoid open in central vein (wavy arrows). B: portal area with portal vein (PV), few inflammatory cell infiltration (I), numerous hepatocytes with vesicular nucleus (arrows), some hepatocytes are binucleated (head arrow), few with vacuolated cytoplasm (curved arrows) and blood sinusoids (wavy arrows). (H\&E X 400)
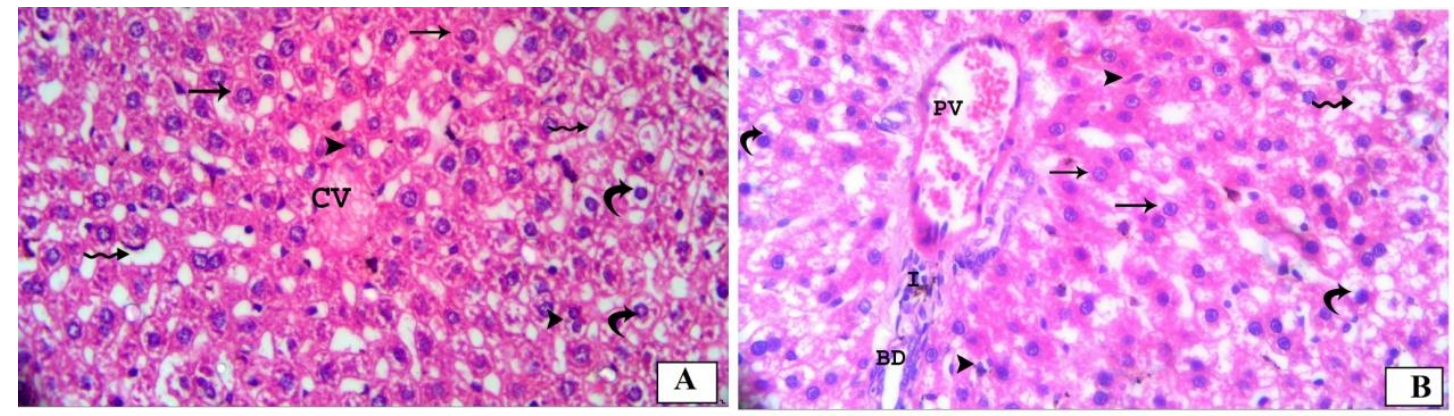

Figure (4) photographs of liver sections from simvastatin - treated group showing, A: congested central vein (CV), wide sinousoid (wavy arrow), hepatocytes with vesicular nucleus (arrow), other hepatocytes are binucleated (head arrow), some with vacuolated cytoplasm (curved arrow). B: congested portal vein (PV), bile duct (BD), wide sinousoid (wavy arrow), inflammatory cell infiltration (I), hepatocytes with vesicular nucleus (arrow), other hepatocytes with pyknotic nucleus (head arrow), some with vacuolated cytoplasm (curved arrow (H\&E X 400)
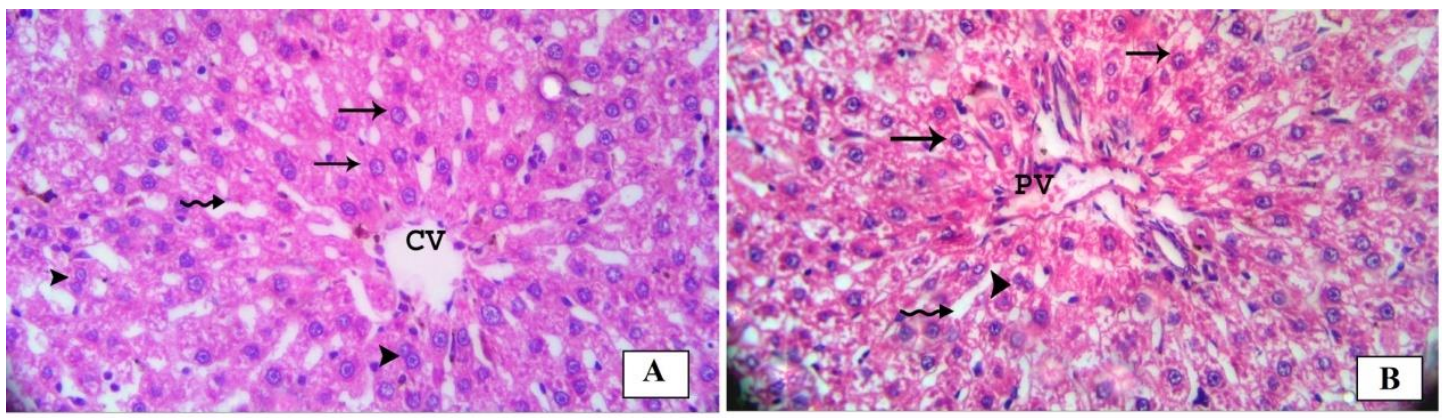

Figure (5): photographs of liver sections from MSCs+Simvastatin- treated group showing, A: nearly normal hepatic tissue the hepatocytes are radiating from the central vein $(\mathrm{CV})$, hepatocytes are with vesicular nuclei and acidophilic cytoplasm (arrow), some hepatocytes are binucleated (head arrows), sinusoids are in between hepatocytic cords (wavy arrow). B: portal area with portal vein (PV), hepatocytes with vesicular nucleus (arrows), some hepatocytes are binucleated (head arrow), and normal blood sinusoids (wavy arrows. (H\&E X 400) 

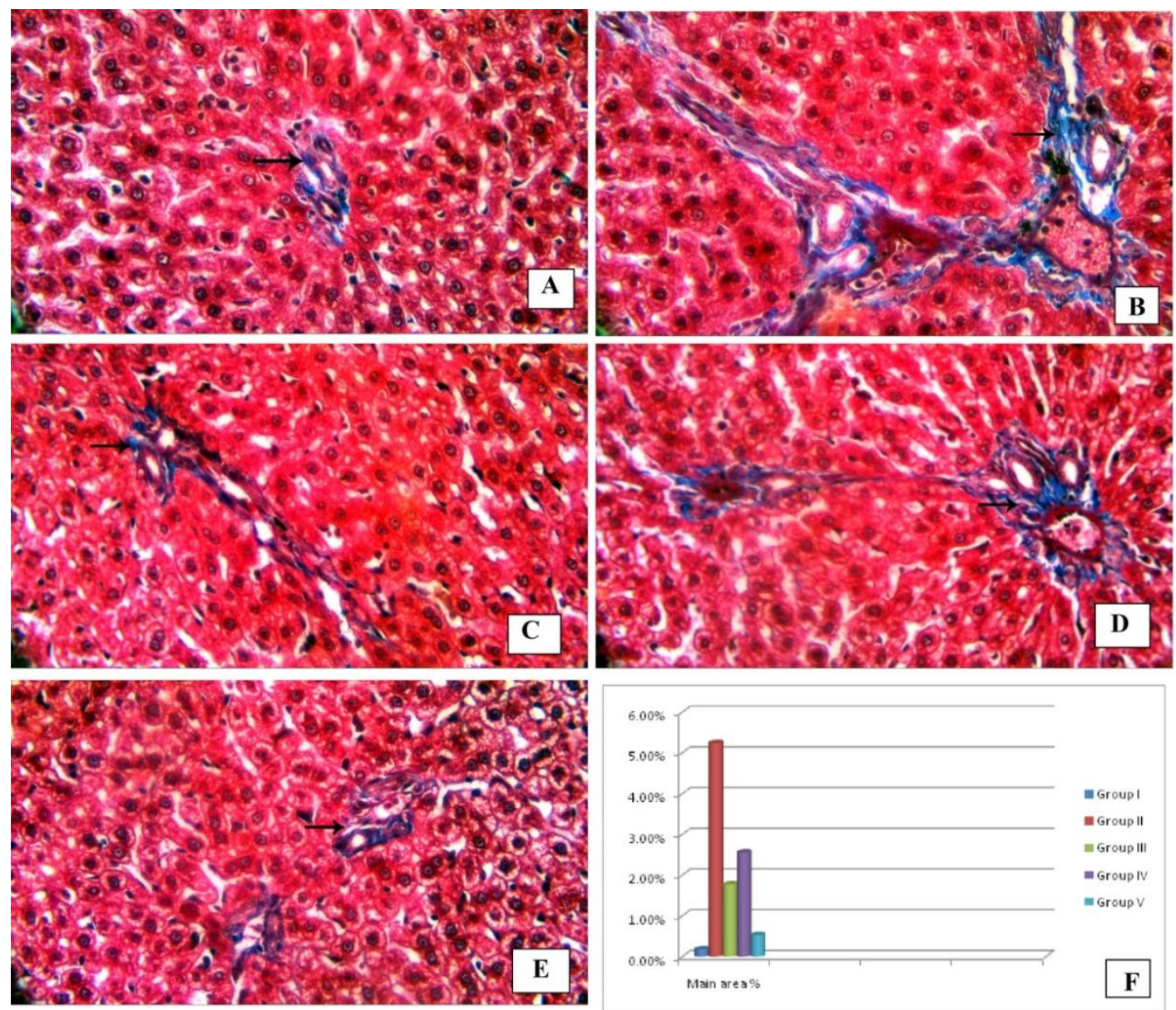

Figure (6): photographs of liver sections stained by Masson trichrome, A: from the control group showing scanty collagen fibers deposition (arrow) around the portal area. B: from the CCL4 group showing marked collagen fibers deposition (arrow) around the portal area. C: from the MSCs-treated group showing mild collagen fibers deposition (arrow) around the portal area. D: from the simvastatin -treated group showing moderate collagen fibers deposition (arrow) around the portal area. E: from the MSCs+ simvastatin -treated group showing few collagen fibers deposition (arrow) around the portal area. (Masson trichrome X400). F: a histogram showing the mean area percent of collagen fibers deposition in groups I, II, III, IV and V. 


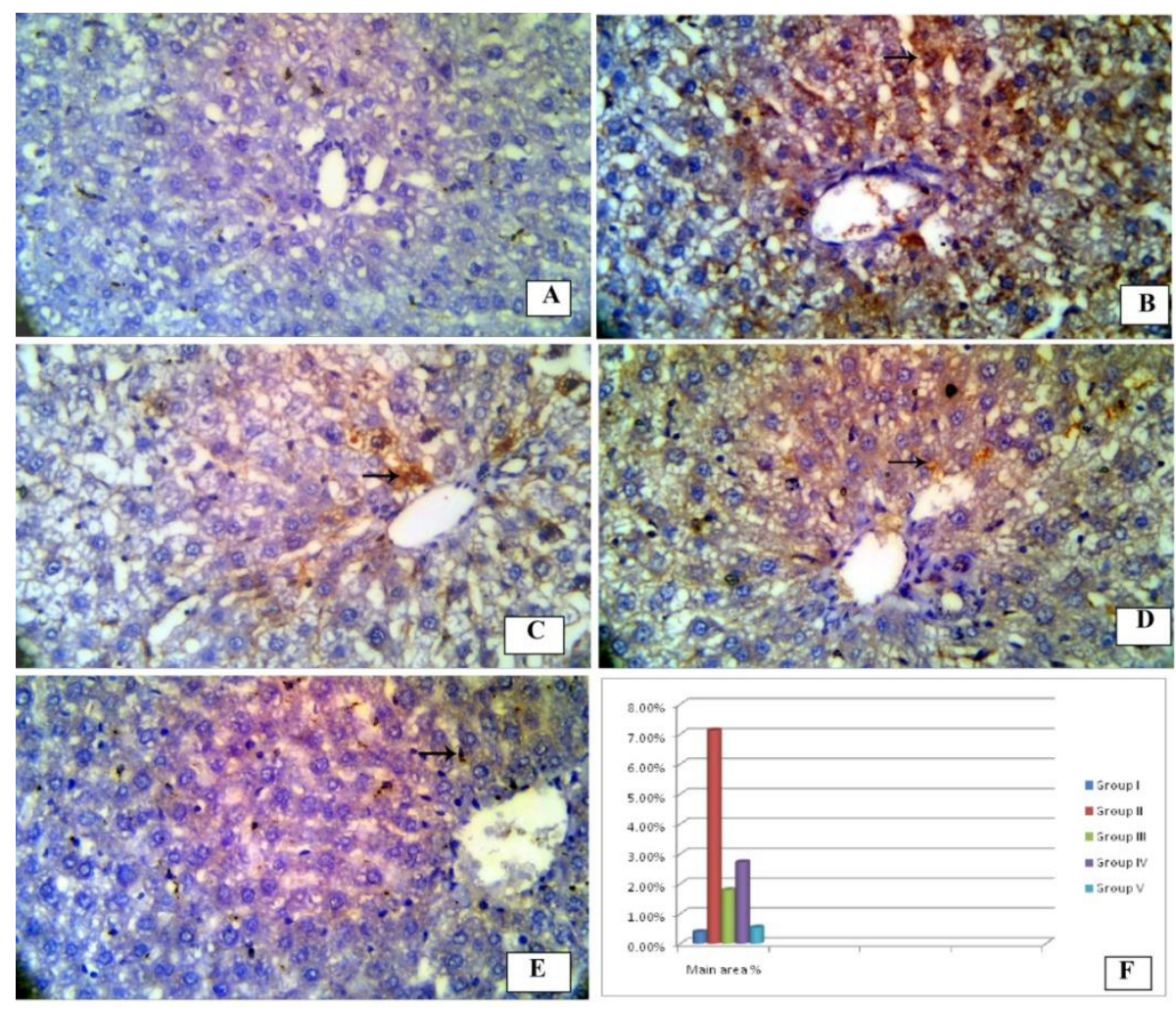

Figure (7): photographs of liver sections stained by TGF b immunostain, A: from the control group showing scanty TGF b immune positive reaction. B: from CCL4 group showing high TGF b immune positive reaction (arrow) in between hepatocytes. C: from MSCs - treated group showing mild TGF b immune positive reaction (arrow) around the central vein. D: from simvastatin - treated group showing mild TGF b immune positive reaction (arrow) in between hepatocytes. E: from MSCs+ simvastatin - treated group showing little TGF b immune positive reaction (arrow) in between hepatocyts. (TGF b immunostaining X 400). F: a histogram showing the mean area percent of TGF $\mathrm{b}$ immune-positive expression in groups I, II, III, IV and V.

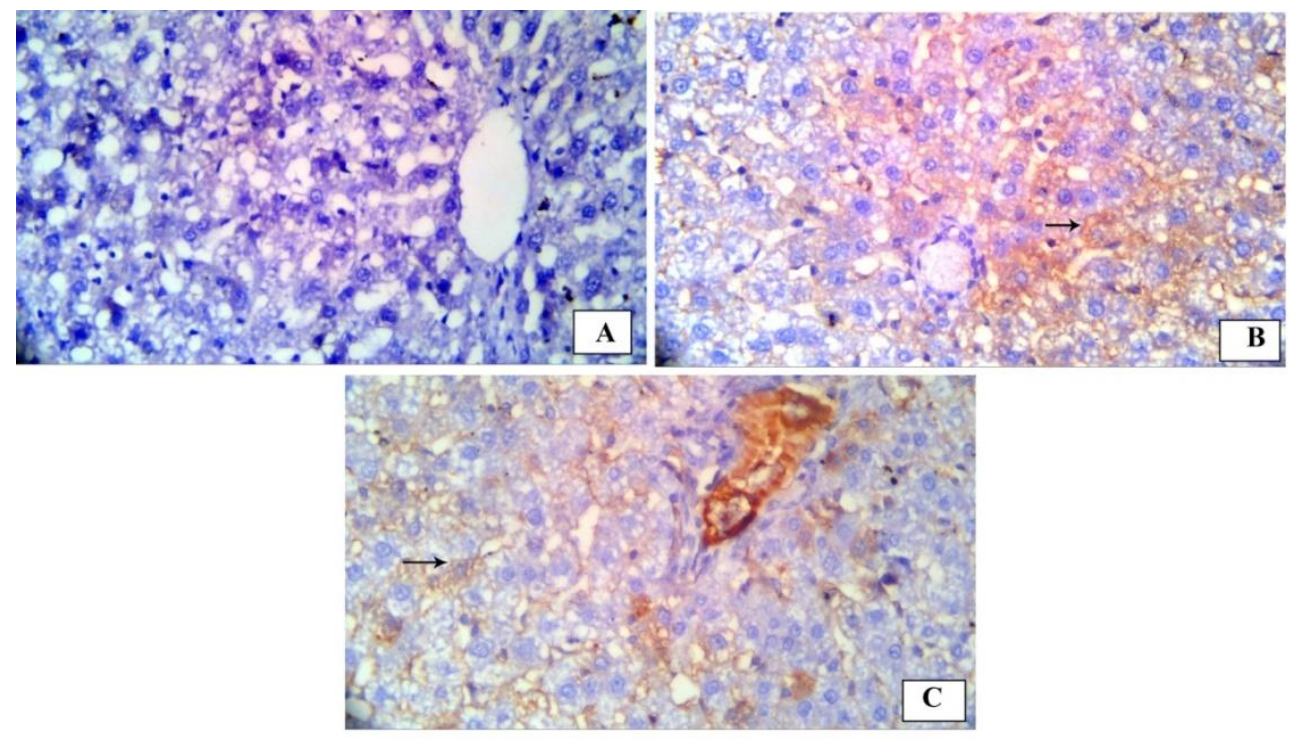

Figure (8): photographs of liver sections stained by CD 105 immunostain, A: from the control group showing negative immune reaction for stem cells. B: from MSCs- treated group showing positive immune reaction for stem cells (arrow). C: from MSCs + simvastatin- treated group showing positive immune reaction for stem cells (arrow). (CD 105 immunostaining X 400) 


\section{Discussion}

Liver is a vital organ in metabolic homeostasis. Fibrosis of hepatic tissue is induced by too much deposition of collagen and ECM components. The mechanism by which CCL4 causes hepatic injury is lipid peroxidation, release of reactive free radical metabolites and disturbance of calcium homeostasis (15).

It has been advised to use Bone marrow mesenchymal stem cells in prevention of liver fibrosis (16). BMSCs are able to motivate healing of hepatic damaged cells and prevent inflammation by secretion of multiple bioactive molecules in addition to its ability to indirectly modulate hepatic satellite cells activation (17).

Statins are along with the mainly talented drugs for treating the portal hypertension and its complications. Statins have vasoprotective, lipid lowering with immumodulatory and anti-inflammatory effects (18). Simvastatin produced improvement in the phenotype of sinusoidal cells, ameliorated the function of liver sinusoidal endothelial cells and has a good role in inhibition of the hepatic stellate cells activity and proliferation (19).
This study is designed to compare between the effect of BM-MSCs, simvastatin or a combination of both on hepatic fibrosis induced by $\mathrm{CCl} 4$ in rats.

In the present study, CCL4 induced liver dysfunction by significant elevation in serum levels of AST, ALT, ALP and significant reduction of albumin level in comparison to those levels in control group. The histopathological examination revealed loss of normal hepatic tissue architectures as there was congested dilated central vein and portal vein, wide congested sinusoid. Most hepatocytes have vacuolated cytoplasm with inflammatory cell infiltration. Moreover, there was a significant elevation in mean area $\%$ of collagen fiber deposition and TGF $b$ immune positive expression. This is in agreement with (20) who found that CCL4 produced sever disturbance of hepatic structures with significant increase in expression of anti $\alpha$-smooth muscle actin and caspase 3 in hepatic stellate cells. Similarly, (21) found that CCL4 caused congestion of portal veins and dilation in bile ducts, hepatocytes with vacuolated cytoplasm and increased in TNF- $\alpha$ and PCNA 
immunopositive reaction. Also (22) found that chronic exposure to CCL4 exceeds the regenerative ability of the liver to return the normal structure

Other study explained the mechanism by which hepatic fibrosis occurs by the imbalance between the transformation of HSCs to myofibroblast like cells and the production of extracellular matrix which increases the expression of TGF- $\beta 1$ (23). Moreover, (24) added that the inflammation have a major role in influence of TGF- $\beta 1$ fibrogenic activity. Another authors described that endoglin and growth factors that are considered major profibrogenic cytokines stimulate change of HSCs to myofibroblasts (25).

The best approach for treatment of liver damage is to produce new hepatocytes replacing injured cells with decrease of deposition of unnecessary ECM. MSCssecreted antifibrogenic molecules that help in prevention of fibrosis (26)

In this study MSCs enhanced improvement in biochemical parameters of liver function by significant reduction in AST, ALT and ALP levels and a significant elevation in serum albumin compared to that in CCL4 group. MSCs moderately preserved the normal histological structure of liver tissue as there were numerous hepatocytes with vesicular nucleus, some hepatocytes were binucleated, with a significant reduction in mean area $\%$ of collagen fibers deposition and TGF $\mathrm{b}$ immune positive expression compared to group II. These results obviously established the antifibrotic effect of BM-MSCs in hepatic fibrosis, this is in the same line with (27) who found that BM-MSCs are able to return normal hepatic histological structure in CCL4 induced liver fibrosis. BM-MSCs improved liver function tests and decreased the toxicity of CCl4.Previous studies reported the therapeutic potential role of MSCs in management of rat liver fibrosis (10\& 28). MSCs have a good role in inhibition of hepatic fibrogenesis by their ability to prevent HSCs conversion from the quiet state to the active one (29).

Other study stated that BM-MSCs had inhibited the profibrotic genes and stimulated the anti-fibrotic hepatic genes activity (30). Other study explained the mechanism by which BM-MSCs could prevent hepatic fibrosis by their ability to secrete vascular endothelial growth factor that has antiapoptotic, antifibrotic activity and regenerated the liver tissue (31) Furthermore, (32) found that MSCs normalize the content of IgA, IgM 
in fibrotic rats and enhanced the activity of glutathione peroxidase in the mitochondria.

Statins show pleiotropic effects in a lot of liver diseases. It inhibited the synthesis of isoprenoid as a consequence of decreased 3hydroxy-3- methyl-glutaryl-coenzyme reductase activity, as the resultant modulated GTPase activity has a main role in the management of the majority of chronic liver diseases (33).

In the present study simvastatin enhanced mild improvement of liver function tests by significant reduction in AST, ALT and ALP levels and a significant elevation in serum albumin compared to that in CCL4 group. Simvastatin mildly improved the histopathological changes of liver tissue, but still there was congested central vein, wide sinusoid, some cells with vacuolated cytoplasm with a significant decrease in mean area percent of collagen fibers and TGF $b$ immune positive expression compared to group II. But the biochemical parameters and histological findings were better in group III in comparison to that in group IV, this means that the antifibrotic effect of MSCs was superior to that of simvastatin in treatment of liver fibrosis.

This result was in the same line with (34) who explained that simvastatin inhibit the endotoxemia caused by chronic liver disease, by prevention of the oxidative stress, improvement of hepatic hemodynamic and decrease of inflammation in $\mathrm{CCl} 4$-cirrhotic rats. Another study stated that Simvastatin decreased hepatic histopathological changes, lower the degree of hepatocellular ballooning and significantly reduced lipid peroxidation. Simvastatin prevents endoplasmic reticulum stress and stimulates the antioxidant enzymes activity in the liver tissue (35).

Similarly, (36) stated that simvastatin is a safe and helpful drug for cirrhotic patients. Also (37) added in a retrospective study on a large number of cirrhotic and pre-cirrhotic patients that statins administration for the reason of lowering serum cholesterol levels was connected with a decrease in the development of hepatic decompensation and hepatocellular carcinoma.

In this study giving both MSCs and simvastatin enhanced marked preservation of normal liver function test as the levels of AST, ALT, ALP and serum albumin was nearly close to that of control group. There was a marked preservation of normal hepatic tissue structure with a significant reduction in mean area percentage of collagen fiber deposition and TGF $b$ immune-positive expression in comparison to groups II, III and IV. These results are in agreement with (10) 
who added that the antifibrotic effect of MSCs and Simvastatin can be recognized by their role in the balance between Matrix metallo-proteinases and their inhibitors which is crucial in fibrogenesis. Another study found that combination of simvastatin and MSCs enhanced strong protecting effects against liver fibrosis by inhibition of transforming growth factor, phospho-Smad3 signaling and $\alpha$-smooth muscle actin in HSCs of cirrhotic liver. Simvastatin may well proceed synergistically with stem cell as a proficient therapeutic strategy for liver cirrhosis (38). A recent study revealed that statins are able to enhance the differentiation of stem cells into a specific lineage. Statins facilitate angiogenesis, cell homing in cell transplantation applications and protect cells against hypoxia (39).

\section{Conclusion}

The effect of BM-MSCs in treatment of liver fibrosis was superior to the effect of Simvastatin. However, the combination of both of them was the best for treatment of the CCl4-induced liver fibrosis.

\section{References}

1- Veidal S, Karsdal M, Vassiliadis E, Nawrocki A, Larsen M, Nguyen Q. et al.: MMP mediated degradation of type VI collagen is highly associated with liver fibrosis identification and validation of a novel biochemical marker assay. PloS one. 2011; 6 (9): 247-53

2- Wu K, Huang R, Wu H, Liu Y, Yang C, Cao S. et al.: Collagen-binding vascular endothelial growth factor attenuates $\mathrm{CCl} 4$-induced liver fibrosis in mice. Mol Med Rep. 2016;14:4680-4686

3- Ahmed A, Mahmoud M, Ouf M and El-Fathaah E: Aminoguanidine potentiates the hepatoprotective effect of silymarin in CCL4 treated rats. Analysis of Hepatology. 2011; 10 (2): 207-215.

4- Li X, Zhang F, Wang D, Li Z, Qin X and Du G: NMR-based metabonomic and quantitative realtime PCR in the profiling of metabolic changes in carbon tetrachloride-induced rat liver injury. J Pharm Biomed Ana. 2014; 15(89):42-49.

5- Ijiri Y, Kato R, Sadamatsu M, Takano M, Okada Y, Tanaka K. et al.: Chronological changes in circulating levels of soluble tumor necrosis factor receptors 1 and 2 in rats with carbon tetrachlorideinduced liver injury. Toxicology. 2014; 0300-483

6- Nunes de Carvalho S, Helal-Neto E, de Andrade DC, Costa Cortez EA, Thole AA, Barja-Fidalgo C. et al.: Bone marrow mononuclear cell transplantation increases metalloproteinase-9 and 13 and decreases tissue inhibitors of metalloproteinase- 1 and 2 expression in the liver of cholestatic rats. Cells Tissues Organs. 2013;198(2):139-48.

7- Mohamed HE, Elswefy SE, Rashed LA, Younis NN, Shaheen MA and Ghanim AM: Bone marrow-derived mesenchymal stem cells effectively regenerate fibrotic liver in bile duct ligation rat model. Exp Biol Med. 2016;241:581e91

8- Okovityĭ SV, Arkad'eva AV, Bezborodkina NN, Sakuta GA, Iaroslavtsev MI, Shulenin SN. et al.: 
New protective effect of simvastatin in rats with experimental steatohepatitis. Eksp Klin Farmakol. 2007;70(3):43-5

9- Abdel Aziz MT, Atta HM, Mahfouz S, Fouad HH, Roshdy NK, Ahmed HH. et al.: Therapeutic potential of bone marrowderived mesenchymal stem cells on experimental liver fibrosis. Clin Biochem 2007;40:893e9.

10- Motawi TM, Atta HM, Sadik NA and Azzam M.: The therapeutic effects of bone marrow-derived mesenchymal stem cells and simvastatin in a rat model of liver fibrosis. Cell Biochem Biophys. 2014;68(1):111-25.

11- Iredale JP, Thompson A and Henderson NC: Extracellular matrix degradation in liver fibrosis: biochemistry and regulation. Biochim Biophys Acta 2013;1832:876e83.

12- Bancroft JD and Layton C: Theory and Practice of HistologicalTtechniques.7 th ed., Churchill Livingstone of El Sevier. Philadelphia. 2013; pp: 172 - 214.

13- Kempinski R, Neubauer K, Poniewierka E, Kaczorowski $\mathrm{M}$ and Halon A: The immunoreactivity of TGF-b1 in non-alcoholic fatty liver disease. Folia Histochem Cytobiol. 2019;57(2):74-83.

14- Sarvandi SS, Joghataei MT, Parivar K, Khosravi M, Sarveazad A and Sanadgol N: In vitro differentiation of rat mesenchymal stem cells to hepatocyte lineage. Iran J Basic Med Sci. 2015; 18(1):89-97.

15- Huang H, Wang Y, Zhang Q, Liu B, Wang F, Li J. et al.: Hepatoprotective effects of baicalein against CCl4- induced acute liver injury in mice. World J Gastroenterol. 2012; 18(45):6605-6613.

16- Cho KA, Ju SY, Cho SJ, Jung YJ, Woo SY, Seoh JY. et al.: Mesenchymal stem cells showed the highest potential forthe regeneration of injured liver tissue compared with othersubpopulations of the bone marrow. Cell Biol Int2009;33:772e7.

17- Zhang $\mathrm{Z}$ and Wang FS: Stem cell therapies for liver failure andcirrhosis. J Hepatol 2013;59:183e5.

18- Tsochatzis EA, Bosch J and Burroughs AK: Liver cirrhosis. Lancet. 2014; 383(9930):1749-61.

19- Janda S, Young A, Fitzgerald JM, Etminan M and Swiston J: The effect of statins on mortality from severe infections and sepsis: a systematic review and meta-analysis. J Crit Care. 2010;25(4):656.e722.

20- Eman Abu-Dief, Doha Mohammed, Nesreen Abd El-Haliem and Ashraf Mohammad El-Badry: Impact of omega-3 fatty acids on evolution of carbon tetrachloride- (CCl4) induced liver cirrhosis in mice : A histological and immunohistochemical study. Egyptian Journal of Histology 2018; 41(1):61-72

21- Hany K. K. Mostafa, Ghada Galal Hamam and Dalia Alaa El-Din Aly El-Waseef: The possible protective role of 6-gingerol on a model of hepatic injury induced by carbon tetrachloride in adult male albino rats: Histological and immunohistochemical study. Egyptian Journal of Histology 2017; 40(4):499-511

22- Eldien, Nashwa A. M. Mostafa and Randa Abd El Aziz Mohamed: Can the liver Regenerative Potential Restore the Normal Liver Structure in Male Mice CCL4 Induced Liver Fibrosis? A Histological and Immunohistochemical Study ejh. 2020; $43(1): 51-62$

23- Al-Rasheed NM, Attia HA, Mohamad RA, AlRasheed NM, Al-Amin MA and Al-Onazi A: Aqueous Date Flesh or Pits Extract Attenuates Liver Fibrosis via Suppression of Hepatic Stellate 
Cell Activation and Reduction of Inflammatory Cytokines, Transforming Growth Factor- beta 1 and Angiogenic Markers in Carbon TetrachlorideIntoxicated Rats. Evid Based Complement Alternat Med. 2015;247357

24- Dooley $\mathrm{S}$ and ten Dijke P: TGF-beta in progression of liver disease. Cell Tissue Res. 2012;347:245-256

25- Puche J, Saiman Y and Friedman S: Hepatic stellate cells and liver fibrosis. Compr. Physiol. 2013;3: 1473-1492.

26- Gholamrezanezhada A, Mirpoura S, Bagherib M, Mohamadnejadb $\mathrm{M}$, Alimoghaddamc $\mathrm{K}$ and Abdolahzadehb L: In vivo tracking of 111In-oxine labeled mesenchymal stem cells following infusion in patients with advanced cirrhosis. Nucl. Med. Biol. 2011;38, 961-967.

27- Khalil MR, El-Demerdash RS, Elminshawy HH, Mehanna ET, Mesbah NM and Abo-Elmatty DM. Therapeutic effect of bone marrow mesenchymal stem cells in a rat model of carbon tetrachloride induced liver fibrosis. Biomed J. 2020:S23194170(20)30048-2. doi: 10.1016/j.bj.2020.04.011.

28- Mohammed NR, Ahmed RH, Roshdy NK, Aref MI, Hassan NM and Saleh HE: Effect of bone marrow-derived mesenchymal stem cells and umbilical cord blood-CD34+ cells on experimental rat liver fibrosis. Int. J. Stem Cell Res. Transplant. 2014; 2 (3): 63-68

29- Zhao DC, Lei J X, Chen R, Yu WH, Zhang XM, Li SN. et al.: Bone marrow-derived mesenchymal stem cells protect against experimental liver fibrosis in rats. World Journal of Gastroenterology. 2005; 11, 3431-3440.

30- Ali G and Masoud MS: Bone marrow cells ameliorate liver fibrosis and express albumin after transplantation in CCl4-induced fibrotic liver. Saudi J. Gastroenterol. 2012; 18, 263-267.

31- Silva GV, Litovsky S, Assad J A, Sousa A L, Martin BJ, Vela D. et al.:Mesenchymal stem cells differentiate into an endothelial phenotype, enhance vascular density, and improve heart function in a canine chronic ischemia model. Circulation 2005; 111, 150-156

32- Anatoliy B, Klimova E, Nikitchenko Y, Davydov V, Zvyagintseva O, Kurguzova N. et al.: Stem cells take part in regulation of prooxidant activity and immunity at liver fibrosis. Am. J. Biomed. Life Sci. 2014; 2 (6-1), 5-12.

33- Schierwagen R, Uschner FE, Magdaleno F, Klein $\mathrm{S}$ and Trebicka $\mathrm{J}$ : Rationale for the use of statins in liver disease. Am J Physiol Gastrointest Liver Physiol. 2017 ;312(5):407-412

34- Tripathi DM, Vilaseca M, Lafoz E, GarciaCalderó H, Viegas Haute G, Fernández-Iglesias A. et al.: Simvastatin Prevents Progression of Acute on Chronic Liver Failure in Rats With Cirrhosis and Portal Hypertension. Gastroenterology. 2018;155(5):1564-1577.

35- Rodrigues G, Moreira AJ, Bona S, Schemitt E, Marroni CA, Di Naso FC. et al.: Simvastatin Reduces Hepatic Oxidative Stress and Endoplasmic Reticulum Stress in Nonalcoholic Steatohepatitis Experimental Model. Oxid Med Cell Longev. 2019:3201873 doi: 10.1155/2019/3201873.

36- Chang FM, Wang YP, Lang HC, Tsai CF, Hou MC, Lee FY. et al.: Statins decrease the risk of decompensation in hepatitis B virus- and hepatitis C virus-related cirrhosis: A population-based study. Hepatology. 2017;66(3):896-907. 
37- Pose E, Trebicka J, Mookerjee RP, Angeli P and Ginès $\mathrm{P}$ : Statins: Old drugs as new therapy for liver diseases? J Hepatol. 2019;70(1):194-202.

38- Jang YO, Kim SH, Cho MY, Kim KS, Park KS, Cha SK. et al.: Synergistic effects of simvastatin and bone marrow-derived mesenchymal stem cells on hepatic fibrosis. Biochem Biophys Res Commun. 2018 26;497(1):264-271.
39- Gorabi AM, Kiaie N, Pirro M, Bianconi V, Jamialahmadi $\mathrm{T}$ and Sahebkar A.: Effects of statins on the biological features of mesenchymal stem cells and therapeutic implications. Heart Fail Rev. 2020 Feb 1. doi: 10.1007/s10741-020-099299

To cite this article: Ali M. Ali, Essam M. Eid, Hanan I. El-Kerdasy, Marian V. Zaki, Naglaa A. Sarg. Comparative Study of the Mesenchymal Stem Cell and Simvastatin in the Treatment of Hepatic Fibrosis in Rats Induced by Carbon Tetrachloride. BMFJ 2022; 39(academic issue):78-95. 